\title{
Time Dependence of the UV Resonance Lines in the Cataclysmic Variable Star VW Hyi
}

\author{
M.T. Özkan ${ }^{1}$, T. Ak ${ }^{2}$, A.T. Saygac ${ }^{2}$, H.H. Esenoglu ${ }^{2}$ and S. Guler ${ }^{2}$ \\ 1 Istanbul University Observatory Research and Application Center, 34452 University- \\ Istanbul, Turkey \\ 2 Istanbul University, Science Faculty, Department of Astronomy and Space Sciences, \\ 34452 University-Istanbul, Turkey
}

\begin{abstract}
In this work, we study orbital variations in the ultraviolet resonance lines of the SU UMa type dwarf nova VW Hyi examining spectra in the data archive of the International Ultraviolet Explorer (IUE). We show that VW Hyi exhibits an orbital and long term variability in terms of UV line profiles and continuum flux at $1450 \AA$ during superoutburst.
\end{abstract}

\section{Introduction}

To learn more about the nature of accretion disks, winds and dwarf novae outbursts, it is useful to study UV observations of cataclysmic variables. These observations reveal that one of the most striking properties of the wind-formed lines observed in dwarf novae is related to the variation of their profiles. There are two types of regular variability in the resonance line profiles. These are dependent on (i) outburst phase, and (ii) orbital phase. It has been found that there are about twenty CVs exhibiting more or less orbital change in their resonance lines (Friedjung et al. 1982, Szkody \& Mateo 1986, Verbunt et al. 1987, Wood et al. 1990, Drew et al. 1991, Wood et al. 1992, Harlaftis et al. 1992, Knigge et al. 1994, van Paradijs et al. 1994, Mansperger et al. 1994, Özkan et al. 1995). They are generally dwarf novae (but one of them, MU Cen, does not show any variation). In addition to the three subgroups of dwarf novae, orbital variations were also discovered in two nova-like systems and one old nova. In this paper, we study the UV line variations associated with the orbit of the SU UMa type dwarf nova VW Hyi. Although VW Hyi has been studied over a large wavelength range through the outburst cycles (Pringle et al. 1987), we believe that it is necessary to consider the wind-formed line profiles in more detail again. We have chosen eight consecutive SWP spectra covering 3.88 orbits during superoutburst. Similarly, a second selection includes a total of ten SWP spectra belonging to three different normal outbursts. We also consider all SWP spectra at quiescence. All spectra are corrected for any wavelength shifts using the centroid of the geocoronal Ly $\alpha$ line.

* Based on the spectra taken from VILSPA IUE database 


\section{Results}

Superoutburst: N V $\lambda 1240$, Si III $\lambda 1300$, Si IV $\lambda 1400$, CIV $\lambda 1550$ profiles and line centroid velocities of three resonance lines examined at increasing orbital phase suggest the presence of regular orbital variations. Although the variability pattern in the CIV, N V lines seems to be similar, Si IV shows somewhat different variability. Continuum fluxes at $1450 \AA$ are also variable during the orbital cycle.

Normal outbursts: Although there is an insufficient phase coverage in the first and third outburst, the data exhibit long-term-scale variations in their UV resonance lines and continuum during 50 and 35 orbits, respectively. On the other hand, the conclusion reached for the second outburst is that the orbital variations in the wind-formed lines and the UV continuum may be present during 2.6 orbits, but the number of spectra is insufficient to confirm the orbital nature of these variations.

Quiescence: Examination of the continuum fluxes at $1450 \AA$ versus JD and orbital phase, reveals two different groups with respect to JD. Every group includes five spectra. No significant variation is found for the UV continuum in the first group. However, the data in the second group lead to a $65 \%$ variation.

In summary, we have determined the variability of the UV resonance lines N V $\lambda 1240$, Si IV $\lambda 1400$, CIV $\lambda 1550$ observed during superoutburst as a function of orbital phase. This confirms a similar result associated with variations of the CIV line in a study of VW Hyi by Verbunt et al. (1987). We detected no significant orbital variation in the normal outburst fluxes compared to the UV superoutburst flux modulation. The same behaviour can be seen in another SU UMa type cataclysmic variable star, $Z$ Cha (Harlaftis et al. 1992). The mean equivalent widths of the UV absorption lines during superoutburst seem to be weaker than during normal outbursts while the continuum flux distribution during superoutburst is stronger than during normal outbursts.

Acknowledgements: We thank the AAVSO and Janet Mattei for obtaining photometric data. M.T. Özkan also thanks the IAU for supporting his travel and accomodation for this colloquium. This work is partly supported by TUBITAK.

\section{References}

Drew J.E., Hoare M.G., Wood J.A., 1991, MNRAS 250, 144

Friedjung M., Andrillat Y., Puget P., 1982, A\&A 114, 351

Harlaftis E.T., et al., 1992, MNRAS 259, 593

Knigge C., et al., 1994, MNRAS 269, 891

Mansperger C.S., et al., 1994, PASP 106, 858

Özkan M.T., et al., 1995, XXIInd General Assembly of the IAU, poster paper (in press)

Pringle J.E., et al., 1987, MNRAS 225, 73

Szkody P., Mateo M., 1986, ApJ 301, 286

van Paradijs J., et al., 1994, MNRAS 267, 465

Verbunt F., et al., 1987, MNRAS 225, 141

Wood J.A., et al., 1990, MNRAS 245, 323

Wood J.A., et al., 1992, MNRAS 255, 237 